\title{
Donación de sangre y medicina transfusional en la prensa española
}

\section{Blood Donation and Transfusion Medicine in the Spanish Press}

\author{
Mariluce Karla Bomfim de Souzaa, Pablo Santoro Domingob \\ a Instituto de Saúde Coletiva, Universidade Federal da Bahia, Brasil \\ b Departamento de Sociología: Metodologia y Teoría, Facultad de Ciencias Políticas y Sociología, Universidad Complutense de \\ Madrid, España
}

\section{Resumen}

Introducción: Los medios de comunicación cumplen un papel fundamental a la hora de fomentar la donación de sangre y de construir los valores y contenidos simbólicos que se asocian a ella. Objetivos: Estudiar las formas en que la prensa española trata la donación de sangre y otras cuestiones relacionadas con la medicina transfusional. Métodos: A partir de una búsqueda realizada en los diarios El País, El Mundo, ABC, La Vanguardia, El Confidencial y eldiario.es, se recopilaron 332 noticias publicadas durante 2016. Se realizó un análisis estadístico por frecuencia simple y un análisis de contenido. Resultados: Las noticias fueron clasificadas por temática, encontrándose que un $45,5 \%$ refería campañas y llamadas para donación; un $26,8 \%$ abordaba cuestiones organizativas relacionadas con los servicios y recursos transfusionales; un $19,6 \%$ trataba sobre investigación médica e innovaciones terapéuticas; y un 11,4\% cuestiones didácticas 0 divulgativas. Además, un $24,4 \%$ de las noticias mencionaba explícitamente significados relacionados con valores como el altruismo, generosidad o solidaridad, mientras que un $29,8 \%$ hacía mención a territorios específicos. Conclusiones: Se identificaron cinco funciones que los diarios cumplirían respecto de la promoción y construcción simbólica de la donación de sangre: apelativa, didáctica, futurista, ideológica y construcción de identidades colectivas.

Palabras clave: Donación de sangre; Hematología; Medicina transfusional; prensa; España.

\begin{abstract}
Introduction: The media play a fundamental role when it comes to promoting blood donation and building the symbolic values and contents associated with it. Objective: The aim of this study was to analyze the ways in which press treats blood donation and other issues related to transfusion medicine, based on an analysis of news published in the main newspapers of Spain during the year 2016. Methods: From a search conducted in the newspapers El Pais, El Mundo, ABC, La Vanguardia, El Confidencial and eldiario.es, 332 news items were selected. A simple frequency statistical analysis was performed, as well as a content analysis. Results: News items were classified by topic, finding that $45.5 \%$ of the news referred campaigns and calls for donation; $26.8 \%$ addressed organizational issues related to transfusion services and resources; $19.6 \%$ dealt with medical research and therapeutic innovations; and $11.4 \%$ addressed didactic or educational issues. In addition, $24.4 \%$ of the news explicitly mentioned values such as altruism, generosity or solidarity, while $29.8 \%$ referred to specific territories or made comparisons between autonomous communities. Conclusions: We identified five functions that newspapers fulfill regarding the promotion and symbolic construction of blood donation: appealing, didactic, futuristic, ideological and construction of collective identities.
\end{abstract}

Key words: Blood donors; Hematology; Transfusion Medicine; Press; Spain. 


\section{Introducción}

a sangre ha llegado a convertirse un elemento esencial para el funcionamiento de los sistemas de salud modernos en diferentes aspectos - desde su uso en transfusiones hasta sus aplicaciones en investigación clínica y biomédica, así como apoyo a diversos procedimientos quirúrgicos y terapéuticos-. A pesar de las tentativas para producir sustitutos artificiales de sangre (Squires, 2002), esta sigue siendo un recurso biológico cuya única fuente se encuentra en la donación individual. De ahí la importancia de contar con políticas públicas adecuadas que estimulen la donación de sangre y garanticen su suministro para atender a la demanda de los centros sanitarios, así como para asegurar la calidad y la seguridad de las muestras y los productos derivados de la sangre.

Según la Organización Mundial de Salud (OMS), en el mundo se extraen anualmente más de 112,5 millones de unidades de sangre, existiendo más de 13.000 centros de donación de sangre en 176 países (Organización Mundial de la Salud, 2017). España, el país en el que se centra el presente estudio, cuenta con un sistema público y no remunerado de donación, sustentado sobre los fundamentos ético-organizativos de voluntariedad, altruismo, anonimato y gratuidad que se corresponden con las recomendaciones de la OMS. Sin embargo, mientras que España es líder mundial en donación de órganos y otros tejidos (tales como donación de médula ósea), no destaca especialmente en sus tasas de donación de sangre (Federación Española de Donantes de Sangre, 2016), lo que hace relevante reflexionar sobre las formas y estrategias para promoverla.

En la promoción de la donación de sangre, al igual que en otras cuestiones de salud pública donde es necesaria la participación de la población o la modificación de sus conductas, los medios de comunicación cumplen un papel central. Clarke y Bins señalan cómo:

Los sentimientos personales, las actitudes, los comportamientos y otros aspectos significativos de la vida de los ciudadanos, incluyendo la salud y la enfermedad, se ven afectados por las historias que se publican en los medios de comunicación. Las imágenes que ofrecen los mass-media condicionan políticas de salud fundamentales, relacionadas, entre otras cosas, con el diagnóstico, el tratamiento, la prevención, la promoción de la salud, la orientación de la investigación básica o el apoyo para determinados servicios" (Clarke y Bins, 2006, p. 39).

Situados en el centro de la esfera pública contemporánea (Habermas, 2010), los medios de comunicación, y particularmente la prensa, son actores centrales en la configuración y framing de los asuntos colectivos, incluidos aquellos relacionados con la salud pública. Por ello se hace necesario estudiar no solo cómo las campañas y acciones llevadas a cabo en los medios influyen sobre los comportamientos de salud de los ciudadanos, sino también cómo se construye simbólicamente un área 0 cuestión en particular (Wakefield, Loken y Hornik, 2010).

Este estudio se alinea así con otras investigaciones sociológicas y de comunicación que analizan los discursos, retóricas y formas de tratamiento que hace la prensa sobre cuestiones de salud (Williams, Kitzinger y Henderson, 2003). Existen diversos estudios que analizan la efectividad de las campañas de promoción de la donación de sangre, entendiéndolas como un determinante más en la motivación de los donantes (Harrington et al, 2007; Gaston Godin et al., 2007; Nilsson Sojka y Sojka, 2008), o que abordan su relación con el conocimiento y actitudes frente a la donación que circulan entre la población de un lugar determinado (Alam y Masalmeh, 2004; Zaller et al., 2005). Sin embargo, aunque en el caso de la donación de órganos sí que hay estudios que analizan simbólicamente los temas abordados en prensa, su contenido discursivo o las narrativas que generan (Feeley y Vincent, 2007; Quick, Kim, y Meyer, 2009; Danet y Medina, 2015; Feeley, Mally, y Covert, 2016), no parecen existir estudios similares que analicen los contenidos simbólicos de la cobertura en prensa sobre donación de sangre, ni en España ni en otros países.

En este sentido, el objetivo del presente estudio fue analizar las formas que los medios de comunicación españoles, y de forma específica los principales periódicos de ámbito nacional, construyen temáticamente los asuntos relacionados con la donación de sangre, así como otras cuestiones relacionadas como las transfusiones sanguíneas o la atención hematológica.

\section{Métodos}

El estudio se realizó en el contexto de una investigación postdoctoral más amplia titulada "Gestión y planificación en salud en el contexto de la regionalización: un análisis a partir de la implementación de las acciones y política de la sangre en el Sistema Único de Salud (Brasil) y Sistema Nacional de Salud (España)", desarrollada por la autora principal en la Universidad Complutense de Madrid.

Para la muestra se tomaron en cuenta cuatro periódicos impresos (El País, El Mundo, ABC y La Vanguardia), así como dos diarios digitales de relevancia (El Confidencial y eldiario.es). Se trata de los cuatro diarios impresos de ámbito nacional con mayor número de lectores y de los dos digitales con mayor número de usuarios (Oficina 
de Justificación de la Difusión, 2016). Se decidió centrar el análisis en un único año, 2016, tanto por razones prácticas como analíticas.

La construcción de la base de datos de noticias fue realizada a través de los buscadores de las páginas web de cada periódico. Se utilizaron los siguientes descriptores para la búsqueda: "donación de sangre"; "donaciones de sangre"; "transfusión de sangre"; "bancos de sangre"; "enfermedades hematológicas"; y "hematología". Para complementar la búsqueda, se recurrió además a los tags con los cuales los diarios categorizan las noticias en sus webs, particularmente "sangre", "donación de sangre" y "transfusiones de sangre".

La búsqueda arrojó 460 noticias publicadas durante 2016, de las cuales se excluyeron posteriormente 128 (ya fuera por duplicación o por tratar solo secundariamente cuestiones sobre donación de sangre o hematología). Así, el corpus de análisis final para este estudio fue de 332 noticias. Con el fin de organizar y procesar los resultados se elaboró una base de datos donde fueron recogidos descriptores básicos de cada noticia (periódico, fecha, título y sección). A partir de la lectura exhaustiva y de la evaluación del contenido de las noticias por parte de los dos autores,se siguió un trabajo de clasificación conjunta y recursiva, mediante el cual se generó un conjunto de siete categorías temáticas que se exponen en la sección de "Resultados". Las categorías generadas no fueron excluyentes, sino que pretendían abarcar la mayor parte posible de los temas y perspectivas abordadas en el corpus analizado, por lo que muchos artículos fueron objeto de una categorización múltiple. Cada una de esas categorías fue analizada tanto cuantitativamente, para conocer su distribución y su presencia estadística dentro de la muestra, como cualitativamente, mediante un análisis de contenido temático (Bardin, 1979; Minayo, 2010). Ambos análisis perseguían identificar los significados representativos del conjunto de las noticias y apuntar las funciones que los periódicos cumplen en la difusión de noticias sobre donación de sangre y hematología.

\section{Resultados}

En cuanto a la distribución por periódico (Gráfico 1), el número de noticias publicadas en La Vanguardia $(n=168$; $51,0 \%$ de la muestra analizada) destaca especialmente, seguido por las informaciones publicadas en eldiario.es $(n=64 ; 19,0 \%)$ y ABC $(n=57 ; 17,0 \%)$. En el caso de El País y El Confidencial, el número de noticias es mínimo, con solamente 3 y 1 noticia respectivamente.

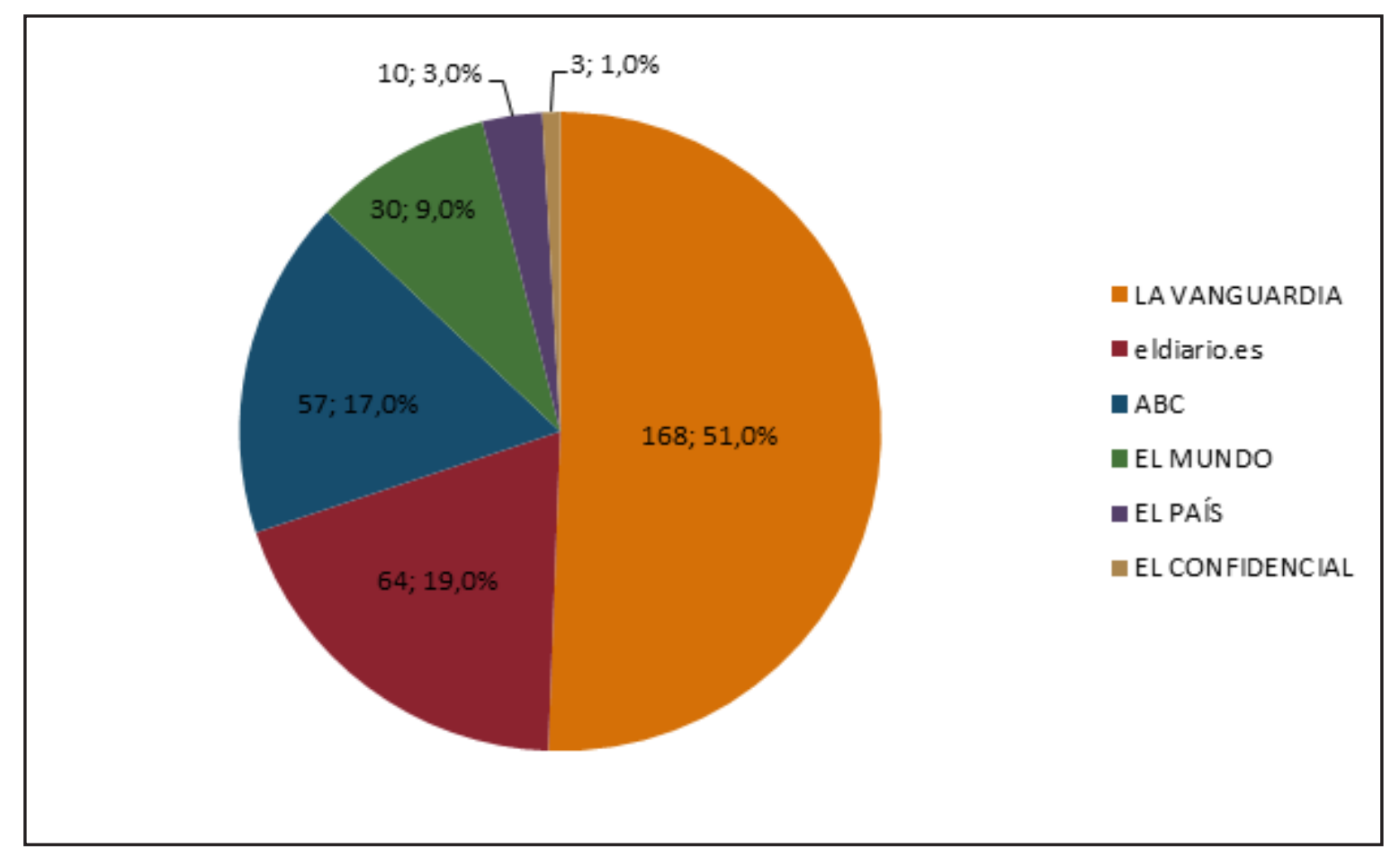

Gráfico 1. Distribución cuantitativa y porcentual de noticias sobre donación de sangre publicadas por periódico, España, 2016. 
Respecto de las secciones del diario donde se publicaron las noticias analizadas (Tabla 1), la mayor parte de la información sobre el tema fue publicada en la sección "Regional" ( $n=164 ; 49,4 \%$ ), seguida por "Sociedad" $(n=119 ; 35,8 \%)$. Apenas un $3,9 \%$ de las noticias $(n=$ 13) se incluyó en la sección "Salud" y el registro también fue pequeño en las secciones "España" $(n=7 ; 2,1 \%)$ e "Internacional" ( $n=5 ; 1,5 \%)$. Esto explicaría también la distribución por periódico, puesto que los tres diarios con mayor número de noticias son también aquellos donde las noticias de ámbito regional o local suelen tener un mayor protagonismo -por ejemplo, La Vanguardia cuenta, además de con su edición estándar, con 8 ediciones parciales para diferentes comarcas catalanas y con 4 ediciones para diferentes comunidades autónomas (Madrid, Andalucía, País Vasco y Comunidad Valenciana)-, mientras que eldiario.es presenta en su web 14 secciones particulares para Comunidades Autónomas, de las que solo están excluidas Asturias, Baleares y La Rioja; por el contrario, El País, que hace ediciones especiales para América Latina o Brasil y tiene una edición diaria en inglés, solamente particulariza su edición para Cataluña.

Tabla 1. Distribución de las noticias analizadas según la sección en los periódicos donde se publicaron, España, 2016.

\begin{tabular}{|l|c|c|c|c|c|c|c|c|}
\hline Sección & La Vanguardia & Eldiario.es & EI Mundo & EI País & ABC & El Confidencial & Total & $\begin{array}{l}\text { \% del } \\
\text { total de } \\
\text { noticias }\end{array}$ \\
\hline Regional & 62 & 56 & 19 & 3 & 22 & 2 & 164 & $49,4 \%$ \\
\hline Sociedad & 97 & 5 & - & - & 17 & - & 119 & $35,8 \%$ \\
\hline Economía & 1 & - & 2 & 1 & 1 & - & 5 & $1,5 \%$ \\
\hline España & 2 & - & - & 3 & 2 & - & 7 & $2,1 \%$ \\
\hline Internacional & 1 & - & - & 2 & 1 & 1 & 5 & $1,5 \%$ \\
\hline Opinión & - & 2 & 1 & - & - & - & 3 & $0,9 \%$ \\
\hline $\begin{array}{l}\text { Cultura/ } \\
\text { espectáculos }\end{array}$ & 2 & 1 & - & - & 3 & - & 6 & $1,8 \%$ \\
\hline Salud & 1 & - & 5 & - & 7 & - & 13 & $3,9 \%$ \\
\hline Tecnología & - & - & - & - & 1 & - & 1 & $0,3 \%$ \\
\hline Ciencia & - & - & - & - & 1 & - & 1 & $0,3 \%$ \\
\hline $\begin{array}{l}\text { Reportaje en } \\
\text { suplemento }\end{array}$ & 1 & - & 2 & - & - & - & 3 & $0,9 \%$ \\
\hline $\begin{array}{l}\text { Deportes y } \\
\text { motor }\end{array}$ & 1 & - & 1 & 1 & 2 & - & 5 & $1,5 \%$ \\
\hline
\end{tabular}


En cuanto a la distribución temporal, el Gráfico 2 muestra la variación cuantitativa de noticias publicadas a lo largo de los meses de 2016. El mayor número de noticias relacionadas con la donación de sangre o temas hematológicos se publicó durante en el mes de junio, con 44 noticias (un 13\% del total), seguido por el mes de diciembre, con 33 noticias (un 10\%). Cabe interpretar que sería el protagonismo de las campañas de fomento de la donación durante el período veraniego y en torno a la Navidad lo que explica esta distribución temporal de las noticias, viéndose influido por el calendario de la Organización Mundial de Salud (que definió el 14 de junio como Día Mundial del Donante de Sangre), así como por los períodos considerados "críticos" por la reducción de los índices de donación, como las vacaciones y los días festivos de final de año (Gráfico 2).

En cuanto a los temas de las noticias, clasificamos las noticias según siete categorías temáticas (TABLA 2), que pasamos a exponer a continuación. La categorización fue múltiple, es decir, una misma noticia puede pertenecer simultáneamente a varias categorías.



Gráfico 2. Variación mensual en cantidad de las noticias sobre donación de sangre y hematología publicadas en cada uno de los periódicos analizados, España, 2016. 
Tabla 2. Frecuencia y porcentaje de noticias publicados por periódico y según categorías temáticas, España, 2016.

\begin{tabular}{|c|c|c|c|c|c|c|c|}
\hline $\begin{array}{l}\text { Categoría } \\
\text { temática }^{1}\end{array}$ & \begin{tabular}{|c|} 
La \\
Vanguardia \\
\end{tabular} & \begin{tabular}{|c|} 
El \\
Mundo
\end{tabular} & Eldiario.es & $A B C$ & El País & EI Confidencial & $\%$ Total $(\mathrm{N})$ \\
\hline $\begin{array}{l}\text { Campañas y } \\
\text { llamadas de } \\
\text { concienciación }\end{array}$ & $53,6 \%$ & $\begin{array}{l}46,7 \% \\
(4)\end{array}$ & $\begin{array}{l}42,2 \% \\
(27)\end{array}$ & $\begin{array}{l}31,6 \% \\
(18)\end{array}$ & $\begin{array}{l}20,0 \% \\
(2)\end{array}$ & $\begin{array}{l}0,0 \% \\
(0)\end{array}$ & $\begin{array}{l}45,5 \% \\
(151)\end{array}$ \\
\hline $\begin{array}{l}\text { Cuestiones } \\
\text { organizativas }\end{array}$ & $\begin{array}{l}34,5 \% \\
(58)\end{array}$ & $\begin{array}{l}26,7 \% \\
(8)\end{array}$ & $\begin{array}{l}23,4 \% \\
(15)\end{array}$ & $\begin{array}{l}7,0 \% \\
(4)\end{array}$ & $\begin{array}{l}30,0 \% \\
(3)\end{array}$ & $\begin{array}{l}33,3 \% \\
(1)\end{array}$ & $\begin{array}{l}26,8 \% \\
(89)\end{array}$ \\
\hline $\begin{array}{l}\text { Investigación } \\
\text { médica y } \\
\text { innovaciones } \\
\text { terapéuticas }\end{array}$ & $\begin{array}{l}26,8 \% \\
(45)\end{array}$ & $\begin{array}{l}16,7 \% \\
(5)\end{array}$ & $\begin{array}{l}1,6 \% \\
(1)\end{array}$ & $\begin{array}{l}19,30 \% \\
(11)\end{array}$ & $\begin{array}{l}20,0 \% \\
(2)\end{array}$ & $\begin{array}{l}33,3 \% \\
(1)\end{array}$ & $\begin{array}{l}19,6 \% \\
(65)\end{array}$ \\
\hline $\begin{array}{l}\text { Cuestiones } \\
\text { didácticas } 0 \\
\text { divulgativas } \\
\end{array}$ & $\begin{array}{l}11,3 \% \\
(19)\end{array}$ & $\begin{array}{l}16,7 \% \\
(5)\end{array}$ & $\begin{array}{l}9,4 \% \\
(6)\end{array}$ & $\begin{array}{l}12,3 \% \\
(7)\end{array}$ & $\begin{array}{l}10,0 \% \\
(1)\end{array}$ & $\begin{array}{l}0,0 \% \\
(0)\end{array}$ & $\begin{array}{l}11,4 \% \\
(38)\end{array}$ \\
\hline Controversias & $\begin{array}{l}4,8 \% \\
(8)\end{array}$ & $\begin{array}{l}10,0 \% \\
(3)\end{array}$ & \begin{tabular}{|l}
$3,1 \%$ \\
$(2)$
\end{tabular} & $\begin{array}{l}17,5 \% \\
(10)\end{array}$ & $\begin{array}{l}40,0 \% \\
(4)\end{array}$ & $\begin{array}{l}66,7 \% \\
(2)\end{array}$ & $\begin{array}{l}8,7 \% \\
(29)\end{array}$ \\
\hline $\begin{array}{l}\text { Epidemiología, } \\
\text { seguridad y } \\
\text { riesgos }\end{array}$ & $\begin{array}{l}4,2 \% \\
(7)\end{array}$ & $\begin{array}{l}13,3 \% \\
(4)\end{array}$ & $\begin{array}{l}12,5 \% \\
(8)\end{array}$ & $\begin{array}{l}14,0 \% \\
(8)\end{array}$ & $\begin{array}{l}10,0 \% \\
(1)\end{array}$ & $\begin{array}{l}0,0 \% \\
(0)\end{array}$ & $\begin{array}{l}8,4 \% \\
(28)\end{array}$ \\
\hline $\begin{array}{l}\text { Accidentes } \\
\text { y desastres } \\
\text { específicos }\end{array}$ & $\begin{array}{l}0,6 \% \\
(1)\end{array}$ & $\begin{array}{l}6,7 \% \\
(2)\end{array}$ & $\begin{array}{l}3,1 \% \\
(2)\end{array}$ & $\begin{array}{l}7,0 \% \\
(4)\end{array}$ & $\begin{array}{l}0,0 \% \\
(0)\end{array}$ & $\begin{array}{l}33,3 \% \\
(1)\end{array}$ & $\begin{array}{l}3,0 \% \\
(10)\end{array}$ \\
\hline
\end{tabular}

${ }^{1}$ Categorias temáticas definidas por los autores a partir de la evaluación del contenido de las noticias. 
El grupo de noticias más numeroso, $151(45,5 \%)$ se refirió a campañas y llamadas para la ciudadanía para la donación, teniendo en cuenta especialmente la reposición de los stocks y la demanda de sangre de determinados grupos sanguíneos. Dentro de ese conjunto, muchas noticias incluían mensajes para la concienciación de las personas en la donación continuada y resaltaban la importancia del "acto", "gesto" y "hábito solidario en su vida cotidiana" para el mantenimiento del sistema público de salud. También se incluyeron en esta categoría noticias que recogen premios o actos de reconocimiento a ciertas personas, colectivos o profesionales de especial relevancia en el ámbito de la donación -por ejemplo, a hermandades de donantes, personas consideradas "grandes donantes" o hematólogos de especial relevancia en el área.

Las noticias que trataban cuestiones organizativas $(n=89 ; 26,8 \%)$ fueron el segundo grupo más numeroso, generalmente en relación con informaciones sobre la mejora de instalaciones en hospitales u otros servicios transfusionales, o sobre la ampliación de la oferta de terapias hematológicas en determinada región o ciudad. Se trata de noticias generalmente de alcance local, y en las que el tema de la donación de sangre, a pesar de tratarse de servicios transfusionales, aparece solo de forma secundaria.

La tercera categoría destacada incluye las noticias que trataban sobre investigaciones biomédicas e innovaciones terapéuticas ( $n=65 ; 19,6 \%$ ), especialmente noticias que presentaban terapias experimentales para el tratamiento y prolongación de la vida de las personas con enfermedades hematológicas. El tema más frecuente en este grupo fue el de las "células madre", retratadas como la principal promesa terapéutica de futuro para enfermedades hematológicas y enfermedades raras. Otras promesas terapéuticas citadas fueron la ingeniería genética para curar la talasemia, el tratamiento CART -receptores quiméricos de antígenos para leucemia linfoblástica-, la creación de glóbulos rojos artificiales, la terapia génica, el tratamiento de la leucemia por activación de la proteína STING, la quimioterapia para proteínas CD33 en la terapéutica de las variantes de mastocitosis o la plataforma integrada para pruebas de NAT como detección del zikavirus.

En cuarto lugar encontramos un grupo de noticias que tratan cuestiones didácticas o divulgativas $(n=38$; $11,4 \%)$, presentando a la población datos sobre la donación de sangre en las diferentes regiones, aclarando los criterios para donar o explicando determinados aspectos de la biología y la terapéutica de la sangre. Son noticias con un tono educativo, que pretenden explicar aspectos más complejos relacionados con la donación de sangre.

En cuanto al grupo de noticias que abordaba controversias y debates sociales $(n=29 ; 8,7 \%)$, durante el año 2016 aparecieron particularmente dos polémicas en la prensa española. En primer lugar, el convenio firmado por la Comunidad de Madrid con la Cruz Roja para que esta organización se ocupara de las unidades móviles de colecta ambulante. Este convenio fue denunciado por algunos actores sociales y partidos políticos, que lo consideraron una forma de privatización encubierta del servicio y de "mercantilización" de la salud, y que durante 2016 llevó al veto de los autobuses de Cruz Roja en algunos municipios madrileños gobernados por la izquierda. Otra polémica recogida en prensa, a partir del ataque terrorista en Orlando (EEUU) a un club gay y las donaciones masivas con las que reaccionó la población, fue el veto aún existente en ciertos países a la donación de sangre por parte de hombres homosexuales, que trajo a discusión los criterios y el posicionamiento de las agencias reguladoras y de los gobiernos. Actualmente existen países que mantienen esta prohibición, mientras que en otros el veto es temporal (variando entre 6 meses y 5 años).

En España, así como en algunos países de América del Sur, la condición sexual del donante no es motivo de discriminación. En el caso español, los requerimientos para donar están legitimados por normativa del Real Decreto 1088/2005, donde solo se considera la buena condición de salud del donador. Tales noticias evidenciaron la contraposición de dos discursos: uno que dice ser protector ("La responsabilidad de la FDA es mantener un alto nivel de seguridad de los productos sanguíneos [...]") y otro considerado discriminatorio, principalmente por los representantes de colectivos LGTB ("La masacre de Orlando levanta el veto de donación de sangre a los homosexuales", El Mundo, 13.06.16). Otras polémicas, por ejemplo las denuncias sobre la discontinuidad y/o suspensión de servicios transfusionales en ciertos territorios o el uso de productos sanguíneos en el dopaje deportivo, tuvieron una presencia mucho menor.

Otros temas fueron más secundarios, pero merecen también ser mencionados. Una categoría con cierta presencia fueron las noticias sobre epidemiología, seguridad y riesgos en la donación y transfusión sanguínea $(n=28$; $8,4 \%$ ), en especial en relación con alertas epidemiológicas de alcance global. Durante el año 2016 destacaron especialmente las alertas en torno al virus zika y la fiebre 
del Nilo, recogiéndose también noticias sobre los riesgos para el stock sanguíneo relacionados con la hepatitis $\mathrm{C}$, el HIV, el ébola o el chagas, en orden decreciente de citación. En particular, la alerta epidemiológica alrededor del virus zika y la inminencia de los Juegos Olímpicos Mundiales de 2016 con sede en Brasil despertaron la atención de los diarios, que reportaron las recomendaciones de la OMS sobre la prohibición (temporal) de donaciones y sobre la contención de transfusiones y las acciones de control epidemiológico ("La OMS recomienda no donar sangre durante un mes tras visitar Brasil", La Vanguardia, 21.06.16).

La última categoría de noticias, poco numerosa pero significativa, se refirió a accidentes y desastres específi$\cos (n=10 ; 3,0 \%)$ sucedidos en algún punto de la geografía y ante los que la población reaccionó acudiendo a donar sangre. El citado atentado de Orlando fue el de mayor repercusión en los periódicos, llegando a provocar "filas en los servicios" para la donación de sangre, pero también se dio noticia, por ejemplo, de un accidente de autobús ocurrido en Freginals (Tarragona), donde murieron trece estudiantes Erasmus y que llevó a que "no pararan de llegar llamadas y gente preguntando si hacía falta donar sangre para atender a las víctimas" (El Diario. es, "Ironías del destino, debemos decir que somos afortunados", 20/03/2016).

El análisis temático de las noticias arrojó también otros resultados de interés, en especial respecto del tratamiento de los valores asociados a la donación y a la dimensión territorial de la cobertura en prensa. En cuanto a los valores, casi una cuarta parte de las noticias ( $n=81 ; 24,4 \%$ ) mencionaba explícitamente el altruismo, generosidad y/o solidaridad, casi unánimemente elegidos como términos significantes para caracterizar el acto de la donación. También llama la atención el peso de la identidad regional y local en muchas noticias, algo que puede relacionarse con la ya citada concentración de noticias en la sección "regional". Muchas noticias se refirieron a ámbitos locales o regionales, y particularmente, a las Comunidades Autónomas. Además, una proporción importante de noticias ofrece comparación de cifras de donación entre las diferentes Comunidades Autónomas ( $n=99 ; 29,8 \%$ ). Esto puede ejemplificarse en fragmentos de dos noticias: "En la región no somos los más ricos en dinero, pero sí somos los más ricos en corazón" ("Extremadura logra más de 2.000 donantes nuevos de sangre", La Vanguardia, 01.10.16), o "Málaga es líder en el turismo, tecnología y cultura, y también queremos que lo sea en solidaridad" ("Centro de Transfusión organiza una colecta", La Vanguardia, 14.12.16).

\section{Discusión}

El análisis del conjunto de noticias publicadas en prensa en 2016 permite señalar algunas cuestiones de interés para reflexionar sobre la comunicación de la donación de sangre y, más en general sobre la cobertura de temas de Salud Pública.

La mayoría de las noticias se ubicó en las secciones "Regional" y "Sociedad". Pocas noticias fueron publicadas en secciones específicas como "salud" o "ciencia". Esto coincide con los resultados del Informe Quiral (Medicina y Salud en la Prensa Diaria, 2017), que identificó que más de la mitad de las noticias sobre salud aparecen en la sección "Sociedad" y apenas un 3,9\% se ubican en secciones específicas, como "Ciencia" o "Salud". Las secciones disponen los contenidos en un mundo simbólico clasificado por el medio de comunicación; esto es, las noticias son situadas en un contexto de producción de información cuyo enfoque es dado por el medio y en un contexto de recepción de la información por el lector (Canel y Sábada, 1999). El "enorme poder de divulgación de la información en salud"(González Borjas, 2004), la diversidad del abordaje y las especificidades del tema pueden constituirse como explicaciones para la publicación de esas en la macroárea llamada "Sociedad".

Avanzando para una comprensión de los significados representativos del conjunto de las noticias, el análisis permite apuntar las siguientes funciones y papeles que los periódicos cumplen en la difusión de noticias sobre donación de sangre y hematología: apelativo; orientativo/didáctico; futurista; ideológico; y de construcción de identidades colectivas.

El tenor apelativo de la mayoría de las noticias encuentra justificación en el propio funcionamiento de los sistemas de colecta de sangre que, al igual que sucede en el caso de órganos y de tejidos, necesitan de acciones de promoción por parte de las autoridades (Mikkelsen, 2007). Este tipo de cobertura surge así como "periodismo de campaña", no obedeciendo a noticias o resultados estrictamente científicos, sino a las ocasiones y a los objetivos de campañas activadas por instituciones públicas 0 privadas, personalidades influyentes, etc. (Thompson y Nelson, 2001). Para Ferrari y Moura (2009) la cobertura periodística de salud también se caracteriza por un atractivo emocional, que va mucho más allá de la simple difusión de innovaciones en el área. Esa función apelativa se ve acentuada por problemas, no particulares de España, que hacen más urgente la necesidad de conseguir nuevos donantes, como el envejecimiento y el cambio generacional del colectivo de donantes o el aumento de los accidentes de tráfico, que acentúa la demanda de los servicios de emergencia y centros quirúrgicos. 
Una segunda función, que se evidencia especialmente en el conjunto de noticias con orientaciones sobre donación de sangre, contenido divulgativo sobre hematología o información sobre innovaciones terapéuticas o sobre seguridad, tiene que ver con el papel orientativo y didáctico de los medios, en tanto medio para "educar" a la población, en una lógica también clásica en el campo de salud pública.

Las noticias sobre investigación médica e innovaciones terapéuticas, que prometen nuevas terapias para curar enfermedades o prolongar la vida de las personas con enfermedades hematológicas, indican la función futurista o de anticipación que cumplen los periódicos y su contribución a generar interés sobre el progreso científico. La mayor parte de las noticias analizadas en este sentido tienen un elemento especulativo que suscita una imaginación sobre el avance de las ciencias biomédicas, a la vez que despierta esperanzas de curación de cara al futuro.Esto parece similar a otras áreas de salud: por ejemplo, un estudio de Oliver y Paúls (2014) sobre información de salud, cuyo objeto era el daño cerebral, mostró que las noticias con un enfoque de difusión científica son predominantes, en comparación con un enfoque político a ciertas iniciativas institucionales (crítica política) o con un enfoque dramático (conciencia social).

Otra función de la prensa es ideológica y de debate político. Diferentes cuestiones polémicas recogidas en el análisis, como la privatización de los servicios sanitarios o la exclusión de la donación de sangre a los hombres homosexuales, vinieron embutidas de carga ideológica,con diferentes posicionamientos de sujetos individuales y colectivos (administradores, partidos políticos, autoridades gubernamentales, agencias reguladoras, movimientos sociales...).

Finalmente, es llamativo cómo las identidades regionales tienen un gran peso en el conjunto de las noticias analizadas. Gran parte de los artículos hacen referencia a poblaciones locales $u$ ofrecen datos comparativos entre las regiones, mencionándose repetidamente el "orgullo" de la población alimentado por los indicadores alcanzados, que indicarían "la generosidad de un pueblo" $y$, también, los esfuerzos organizacionales para la manutención o destaque en los datos numéricos de donación. Este grupo de noticias se solapa además con las referencias sobre solidaridad, generosidad y/o altruismo. También los desastres y catástrofes, considerados en la categoría sobre accidentes, generan conmoción y estimulan acciones solidarias; sobre eso, se constata que las "donaciones de sangre siempre aumentan después de los desastres", lo que ha llevado a hablar de una "economía moral de la donación"(Healy, 2006).
En esa confluencia de los significados de la identidad territorial y el altruismo, se puede observar, por tanto, la última de las funciones identificada en la cobertura de prensa: la construcción simbólica de identidades colectivas en torno a la donación de sangre. El estudio clásico de Titmuss (1971) sobre los sistemas estatales de donación de sangre desarrollados en los países europeos a partir de los años 50 enfatizaba, mediante la teoría de la reciprocidad del "don" de Marcel Mauss, cómo la donación altruista pasó a ser considerada como un acto de solidaridad social y de cohesión nacional (Titmuss, 1971). De acuerdo con Titmuss, los mecanismos públicos de redistribución de sangre, donada por los ciudadanos bajo condiciones de desinterés, gratuidad y anonimato, garantizaban al mismo tiempo la seguridad y calidad de la sangre colectada y su contribución a una lógica moral más amplia de solidaridad, reciprocidad y unión social. La donación no solo servía para garantizar la salud de la población, sino también para contribuir a su cohesión social y política - para construir una nación y una sociedad solidaria, fomentando una identidad colectiva que se vinculaba mediante la lógica de reciprocidad que generaba la donación altruista.

Un amplio conjunto de las noticias publicadas en España sobre donación de sangre durante 2016 puede igualmente ser interpretado a partir de ese prisma. Pero a diferencia de lo que ocurre con la donación de órganos, que históricamente se construyó en España desde la referencia a la identidad nacional (Danet y Medina Domenech, 2013) y desde la perspectiva del conjunto del Estado, no parece en cambio existir una identidad nacional ligada a la sangre. Solamente 3 de las noticias analizadas mencionan los datos del total de España o se refieren al territorio nacional en su conjunto. Al contrario, la donación de sangre aparece repetidamente retratada en la prensa como un mecanismo de solidaridad y de cohesión vinculado exclusivamente a territorios regionales, en especial a identidades locales (pueblos, zonas), autonómicas o incluso nacionalistas. Un ejemplo es especialmente ilustrativo a ese respecto: la llamada a los ciudadanos catalanes a donar realizada por el entonces presidente de la Generalitat, Carles Puigdemont, con ocasión del Día Mundial del Donante, e interpretada en su discurso como parte del propio proceso de reivindicación independentista en que la región está envuelta desde 2009: "La participación coral en la cohesión de la sociedad es absolutamente indispensable para hacer un país nuevo. No se acaban los países ni se consiguen hacer con los textos legales, que son muy importantes. Para poder ser un país avanzado, ha de ser un país cohesionado; y solo puede serlo si las personas nos comprometemos en nuestra pe- 
queña valía de esta cadena de construcción de un país que es la solidaridad entre las personas" (recogido en El Mundo, 14.06.16).

\section{Conclusiones}

En el artículo hemos querido aproximarnos a los significados de la donación de sangre que se construyen a través de su cobertura en prensa.Se ha mostrado la vinculación de la información publicada con las campañas institucionales de fomento de la donación, pero también se ha destacado la importancia de otros contenidos simbólicos, como la construcción de expectativas futuras en torno a las innovaciones biomédicas, la alerta sobre riesgos de salud pública o los debates políticos e ideológicos que pueden generarse en torno a las formas de organizar el acceso a la donación o la gestión pública de la misma.

El análisis ha mostrado también la relevancia de los valores asociados a la donación, como la solidaridad y el altruismo, y su relación directa con la construcción de identidades colectivas, que, en el caso español, apuntan más bien hacia identidades territoriales parciales antes que a la identidad nacional en su conjunto; el contraste que se produce a este respecto con la donación de órganos (donde el orgullo de ser líderes mundiales apuntala una lógica de construcción de una identidad colectiva española, nacional) resulta ilustrativo y debería ser motivo de reflexión.

Para la posible continuación de esta línea de investigación, sería interesante ampliar el marco temporal para facilitar la comparación de diferentes años e incluir otros medios de comunicación que aquí no se han tenido en cuenta, y muy particularmente acercarse a las redes sociales, que cobran cada vez más importancia en la difusión de campañas y como medio de información de la ciudadanía. También se presenta como perspectiva para nuevos estudios el análisis y la discusión de los acontecimientos y eventos políticos relacionados con la donación de sangre en otros países del mundo. El protagonismo de las campañas, evidenciado en este estudio, puede estimular nuevas investigaciones sobre la efectividad de las diferentes acciones y estrategias de promoción de la donación de sangre y de la Salud Pública en general.

\section{Referencias Bibliográficas}

Alam, M., y Masalmeh, B. E. D. (2004). Knowledge, attitudes and practices regarding blood donation among the Saudi population. Saudi Medical Journal, 25(3), 318-321. Recuperado de https://pdfs. semanticscholar.org/bb05/9541d0813590fb5c29ff2e8 e51a5d743c0ed.pdf
Bardin, L. (1979). Análise de conteúdo. Lisboa, Portugal: Edições 70.

Canel, M. J., y Sádaba, T. (1999). La investigación académica sobre las actitudes profesionales de los periodistas. Una descripción del estado de la cuestión. Comunicación y Sociedad, 12(2), 9-32.

Danet, A. D. (2013). Los trasplantes de órganos en España. Cuerpo, emociones e identidad nacional en la prensa contemporánea (19001975) (Tesis doctoral, Universidad de Granada, España). Recuperado de https://hera.ugr.es/ tesisugr/22412311.pdf

Danet, A., y Medina Domenech, R. (2015). A tale of two countries: Narratives of hearts, patients and doctors in the Spanish press. Public Understanding of Science, 24(6), 641-657.

Federación Española de Donantes de Sangre. (2016). Datos estadísticos sobre donación de sangre en España 2016. Recuperado de http://www. donantesdesangre.net/papelfenadose4.htm.

Feeley, T. H., O'Mally, A. K., \& Covert, J. M. (2016). A Content Analysis of Organ Donation Stories Printed in U.S. Newspapers: Application of Newsworthiness, Health Communication, 31(4), 495-503. doi: 10.1080/10410236.2014.973549

Feeley, T. y Vincent III, D. (2007). How organ donation is represented in newspaper articles in the United States. Health Communication, 21 (2), 125-131. doi: 10.1080/10410230701307022

Ferrari A. P., y Moura, D. O. (2009). Consumo, cidadania e direito à saúde - a imprensa e o cidadão quando 0 assunto é o risco sanitário. Revista Interin, 8(2), 2009.

Gaston Godin, G., Conner, M., Sheeran, P., BélangerGravel, A., yGermain, M. (2007). Determinants of repeated blood donation among new and experienced blood donors. Transfusion, 47(9), 1607-1615. doi: 10.1111/j.1537-2995.2007.01331.x

González Borjas, A. (2004). Salud, información periodística especializada en alza. Ámbitos. Revista Internacional de Comunicación, 11-12, 301-310. Recuperado de https://idus. us.es/bitstream/handle/11441/67675/revistacomunicacion-ambitos-11-12_287-296. pdf?sequence=1\&isAllowed=y

Habermas, J. (1982). Historia y crítica de la opinión pública. Barcelona, España: Gustavo Gili. 
Harrington, M., Sweeney, M. R., Bailie, K., Morris, K., Kennedy, A., Boilson, A. J., ... Staines, A. (2007). What would encourage blood donation in Ireland? VoxSanguinis, 92(4), 361-367. doi: 10.1111/j.14230410.2007.00893.x

Healy, K. J. (2006). Last best gifts: altruism and the market for human blood and organs. Illinois, EUA: University de Chicago.

Medicina y Salud en la Prensa Diaria. (2017, Julio 08). Informe Quiral 1997-2006. Análisis de 10 años de información sanitaria en El País, El Mundo, ABC, La Vanguardia y El Periódico de Catalunya. Observatorio de la Comunicación Científica Universitat Pompeu Fabra. Recuperado de https://www.upf.edu/pcstacademy/_docs/Quiral10. pdf.

Mikkelsen, N. (2007). Promotion Activities in Blood Donation. ISBT Science Series, 2(2), 92-97.

Minayo, M. C. S. (2010). O desafio do conhecimento: pesquisa qualitativa em saúde. $12^{\mathrm{a}}$ ed. São Paulo, Brasil: Hucitec.

Clarke, J.N., y Bins, J. (2006). The portrayal of heart disease in mass print magazines, 1991-2001. Health Communication, 19(1), 39-48. doi: 10.1207/ s15327027hc1901_5

Nilsson Sojka, B., y Sojka, P. (2008). The blood donation experience: self-reported motives and obstacles for donating blood. Vox Sanguinis, 94(1), 56-63. doi: 10.1111/j.1423-0410.2007.00990.x

Oficina de Justificación de la Difusión. (2016). Datos certificados sobre tirada y difusión de diarios. Recuperado de http://www.introl.es/medioscontrolados/.

Oliver S. E., y Paúls, B. G. (2014). El encuadre de los temas de salud: cobertura en prensa escrita del daño cerebral adquirido. Ámbitos - Revista Internacional de Comunicación, 26, 1-24. Recuperado de https:// www.printfriendly.com/p/g/7NYMSx

Organización Mundial de la Salud. (2017). Disponibilidad y seguridad de la sangre a nivel mundial. Disponible en http://www.who.int/mediacentre/ factsheets/fs279/es. Recuperado de 01 de agosto de 2017.

Quick, B. L., Kim, D. K., y Meyer, K. (2009). A 15-year review of $A B C, C B S$, and $N B C$ news coverage of organ donation: implications for organ donation campaigns. Health Communication, 24(2), 137145. doi: $10.1080 / 10410230802676516$
Squires, J. E. (2002). Artificial Blood. Science, 295, 1002-1005.

Thompson, R. A., y Nelson, C. A. (2001). Developmental science and the media: Early brain development. American Psychologist, 56(1), 5-15.doi: 10.1037//0003-066X.56.1.5

Titmuss, R. M. (1971). The Gift Relationship. From Human Blood to Social Policy. Harmondsworth, Londres: Penguin Books.

Wakefield, M. A., Loken, B., y Hornik, R. C. (2010). Use of mass media campaigns to change health behavior. The Lancet, 376, 1261-71.doi:10.1016/S01406736(10)60809-4

Williams, C., Kitzinger, J., y Henderson, L. (2003). Envisaging the embryo in stem cell research: rhetorical strategies and media reporting of the ethical debates. Sociology of Health \& IIIness, 25(7), 793-814.doi: 10.1046/j.1467-9566.2003.00370.x

Zaller, N., Nelson, K. E., Ness, P., Wen, G., Bai, X. y Shan, H. (2005). Knowledge, attitude and practice survey regarding blood donation in a Northwestern 